\title{
The Effect of Measurement Error on the Power of X Charts with Known Coefficient of variation
}

\author{
Bhayare Unnati $^{{ }^{*}}$ and Singh Jais Raj ${ }^{2}$ \\ ${ }^{1 *}$ School of Studies in Statistics, Vikram University, Ujjain, 456010, India \\ ${ }^{2}$ School of Studies in Statistics, Vikram University, Ujjain, 456010, India \\ *Corresponding Author: unnati.b80@gmail.com
}

Available online at: www.isroset.org

Received $12^{\text {th }}$ Aug 2017, Revised $30^{\text {th }}$ Aug 2017, Accepted $22^{\text {th }}$ Set 2017 , Online $30^{\text {th }}$ Oct 2017

\begin{abstract}
Measurement error often exists in quality control applications. This error has an adverse effect on the $\overline{\mathrm{X}}$ chart's performance. Measurement error (ME) is a source of variation that may considerably affect the performance of control charts applied within a statistical process control scheme. While the consequences of ME on the actual performance of various control charts has been studied in recent publications, the more important inverse problem of how to specify ME requirements to achieve desirable control chart performance characteristics has not been addressed in the literature. In this paper, we develop guidelines regarding the formulation of power function to distribution-related ME characteristics with known coefficient of variation and investigate the effect of $\mathrm{ME}$ and $\mathrm{cv}$ on power of mean chart.
\end{abstract}

Keywords: Mean chart, Power, Measurement Error, Coefficient of Variation

\section{INTRODUCTION}

In any production process, some variations in product quality are unavoidable. These variations, which in general are attributed due to chance causes or assignable causes, may result in an unacceptable level of process mean, process variance or both. Variations due to chance causes are random in nature. Their occurrence is not predictable and they do not occur in repetitive cycles. The resulting variations are rarely produced by only one cause; rather, they are mostly due to a number of interactive causes. Some typical chance causes of variations are: slight variations in raw materials, slight vibration of machine, lack of operator perfection in reading measurements and setting controls. Variations due to assignable causes are non-random in nature. Their occurrence is predictable and repetitive, and they may result in a large amount of variations in product quality. Some typical assignable causes of variations are: defective raw materials, faulty setup, untrained operator, cumulative effects of heat, vibration, shock, etc. Generally, quality control procedures are used to maintain production in such a way that the output of the process meets the required production specifications. Traditionally, the specification limits are set based on the knowledge of variability due to chance effects. During the production process, variability due to assignable cause may drive the process into an out-of-control state. In controlling a process, the objective is, to detect the shift in the process mean and eliminate the assignable cause. This is facilitated by the use of control charts. When the product quality is measured on a continuous scale, commonly used statistical quality control charts for controlling the process means are the X-chart, the Ychart with warning limits, and the cumulative sum (cusum) chart. To use a control chart, the user must specify a sample size, a sampling interval and the control limits or the critical region for the chart. Selection of these parameters is called the design of the control chart.

\section{POWER OF $\bar{X}$-CHART IN THE PRESENCE OF MEASUREMENT ERROR}

Suppose that the process is subject to measurement error. Let $\mathrm{X}_{\mathrm{i}}(\mathrm{i}=1,2, \ldots, \mathrm{n})$ be a random sample of the "true" values for the process with $\mathrm{X}_{\mathrm{i}} \sim \mathrm{N}\left(\mu, \sigma_{\mathrm{p}}^{2}\right)$. However, due to measurement error, suppose we actually observe $\mathrm{X}_{\mathrm{i}}=\mathrm{X}_{\mathrm{i}}+\varepsilon_{\mathrm{i}}$ where $\left(\varepsilon_{\mathrm{i}} \sim \mathrm{N}\left(0, \sigma_{\mathrm{e}}^{2}\right)\right)$ represent the measurement error. We further assume that $\mathrm{X}_{\mathrm{i}}, \mathrm{X}_{\mathrm{i}}$ and $\varepsilon_{\mathrm{i}}$ are all independent of each other. The power function should be adjusted to account for measurement error in the case $\sigma_{\mathrm{x}}^{2}=\sigma_{\mathrm{p}}^{2}+\sigma_{\mathrm{e}}^{2}$. $\sigma_{\mathrm{x}}^{2}$ is 
commonly referred to as the inter-individual variance while $\sigma_{\mathrm{e}}^{2}$ is the intra-individual variance. Set $\mathrm{r}^{2}=\sigma_{\mathrm{p}}^{2} / \sigma_{\mathrm{e}}^{2}$ and $\rho^{2}=\sigma_{p}^{2} / \sigma_{x}^{2}$; this ratio provides a standardized measure of the size of measurement error. In practice the value of $r^{2}$ depends highly both on the true values and measurement methodology. We assume that $\mathrm{r}^{2}$ can take a wide span of values. The relation between the size of measurement error $r$ and correlation coefficient $\rho$ is obtained as

$$
\rho=\frac{\mathrm{r}}{\sqrt{1+\mathrm{r}^{2}}}
$$

Thus the data used for establishing the power function of $\bar{X}$-charts comes from a process i.e. $N\left(\mu, \sigma_{p}^{2}+\sigma_{e}^{2}\right)$. Following Searls (1964), we define a class of estimators for measurement error as $\overline{\mathrm{X}}^{\prime}=\omega \sum_{\mathrm{j}=1}^{\mathrm{n}} \mathrm{x}_{\mathrm{j}}$. After some simplification the MSE of observed mean $\overline{\mathrm{X}}^{\prime}$ is given by,

$$
\operatorname{MSE}\left(\bar{x}^{\prime}\right)=\mu^{2}\left[\omega^{2} n^{2}\left(1+\frac{v^{2}}{n \rho^{2}}\right)-2 \omega n+1\right],
$$

where $\mathrm{v}=\sigma_{\mathrm{p}} / \mu$. The MSE $\left(\overline{\mathrm{x}}^{\prime}\right)$ of equation (2) is minimum when $\mathrm{w}=1 /\left(\left(\mathrm{v}^{2} / \rho^{2}\right)+\mathrm{n}\right)$. Substituting the value of $w$ in equation (2) which result the minimum $\operatorname{MSE}\left(\overline{\mathrm{X}}^{\prime}\right)$ as,

$$
\operatorname{Min} . \operatorname{MSE}\left(\bar{x}^{\prime}\right)=\frac{\sigma_{p}^{2}}{\left(n \rho^{2}+v^{2}\right)}
$$

The

observed density function of the sample mean $\overline{\mathrm{X}}^{\prime}$ is given by.

$$
\mathrm{f}\left(\overline{\mathrm{x}}^{\prime}\right)=\frac{\sqrt{\mathrm{n} \rho^{2}+\mathrm{v}^{2}}}{\sigma_{\mathrm{p}}}\left[\phi\left(\frac{\overline{\mathrm{x}}^{\prime}-\mu}{\sigma_{\mathrm{p}} / \sqrt{\mathrm{n} \rho^{2}+\mathrm{v}^{2}}}\right)\right] .
$$

If we act

as $\mathrm{X}_{\mathrm{i}}$ are not subject to measurement error and obtain the power function based on the actual observations the resulting value is -

$$
\mathrm{P}_{\overline{\mathrm{x}}^{\prime}}=\operatorname{Pr}\left[\overline{\mathrm{x}}^{\prime} \geq \mu+3 \sqrt{\sigma_{\mathrm{p}}^{2}} / \mathrm{n}\right]+\operatorname{Pr}\left[\overline{\mathrm{x}}^{\prime} \leq \mu-3 \sqrt{\sigma_{\mathrm{p}}^{2} / \mathrm{n}}\right] .
$$

process shift, the data is assumed to come from an $\mathrm{N}\left(\mu^{\prime}, \sigma_{\mathrm{p}}^{2} /\left(n \rho^{2}+\mathrm{v}^{2}\right)\right)$ population.

Converting to a standard normal distribution, we have

$$
Z=\frac{\bar{x}^{\prime}-\mu^{\prime}}{\sqrt{\sigma_{p}^{2} /\left(n \rho^{2}+v^{2}\right)}} \text {. }
$$

Using the new variable, equation (5) can be expressed:

$$
\mathrm{P}_{\overline{\mathrm{x}}^{\prime}}=\operatorname{Pr}\left[\overline{\mathrm{x}}^{\prime}-\mu^{\prime} \geq \mu-\mu^{\prime}+3 \sqrt{\sigma_{\mathrm{p}}^{2} / \mathrm{n}}\right]+\operatorname{Pr}\left[\overline{\mathrm{x}}^{\prime}-\mu^{\prime} \leq \mu-\mu^{\prime}-3 \sqrt{\sigma_{\mathrm{p}}^{2} / \mathrm{n}}\right]
$$


$\mathrm{P}_{\overline{\mathrm{x}}^{\prime}}=\operatorname{Pr}\left[\frac{\overline{\mathrm{x}}^{\prime}-\mu^{\prime}}{\sqrt{\frac{\sigma_{\mathrm{p}}^{2}}{\left(n \rho^{2}+\mathrm{v}^{2}\right)}}} \geq \frac{\mu-\mu^{\prime}}{\sqrt{\frac{\sigma_{\mathrm{p}}^{2}}{\left(n \rho^{2}+\mathrm{v}^{2}\right)}}}+\frac{3 \sqrt{\sigma_{\mathrm{p}}^{2} / \mathrm{n}}}{\sqrt{\frac{\sigma_{\mathrm{p}}^{2}}{\left(n \rho^{2}+\mathrm{v}^{2}\right)}}}\right]$

$+\operatorname{Pr}\left[\frac{\bar{x}^{\prime}-\mu^{\prime}}{\sqrt{\frac{\sigma_{p}^{2}}{\left(n \rho^{2}+v^{2}\right)}}} \leq \frac{\mu-\mu^{\prime}}{\sqrt{\frac{\sigma_{p}^{2}}{\left(n \rho^{2}+v^{2}\right)}}}-\frac{3 \sqrt{\sigma_{p}^{2} / n}}{\sqrt{\frac{\sigma_{p}^{2}}{\left(n \rho^{2}+v^{2}\right)}}}\right]$

$=\operatorname{Pr}\left[Z \geq-d \sqrt{n \rho^{2}+v^{2}}+3 \sqrt{\frac{n \rho^{2}+v^{2}}{n}}\right]+\operatorname{Pr}\left[Z \leq-d \sqrt{n \rho^{2}+v^{2}}-3 \sqrt{\frac{n \rho^{2}+v^{2}}{n}}\right]$

where

$\mathrm{d}=\frac{\mu^{\prime}-\mu}{\sigma_{\mathrm{p}}}$

$=\operatorname{Pr}\left[\mathrm{Z} \geq \sqrt{\frac{\mathrm{n} \rho^{2}+\mathrm{v}^{2}}{\mathrm{n}}}(3-\mathrm{d} \sqrt{\mathrm{n}})\right]+\operatorname{Pr}\left[\mathrm{Z} \leq \sqrt{\frac{\mathrm{n} \rho^{2}+\mathrm{v}^{2}}{\mathrm{n}}}(-3-\mathrm{d} \sqrt{\mathrm{n}})\right]$

$=\Phi\left[\sqrt{\frac{n \rho^{2}+v^{2}}{n}}(3-d \sqrt{n})\right]+\Phi\left[\sqrt{\frac{n \rho^{2}+v^{2}}{n}}(-3-d \sqrt{n})\right]$,

The equation (8) is the power function under measurement error with known cv. For known cv and $r$ these adjusted power function can be readily computed.

\section{NUMERICAL ILLUSTRATION AND CONCLUSION}

When adjusting the measurement error the parametric power function will be calculated by equation (8) for some chosen values of $\mathrm{d}, \mathrm{r}=\infty, 2,4,6$ and $\mathrm{v}=0,2,4,6$ and $\mathrm{n}=5,7$ and given in Table 1 and 2. To give visual comparison the power curves with known cv have been drawn in Figure 1 to Figure 8. The absence of measurement error is equivalent to $r=\infty$. As $r$ (size of the measurement error) increases from $\infty$ to four the power function for $v=0, n=5$ increases by two times for $d=1$.4. If measurement error of this size is ignored, the unadjusted power curves will give the erroneous impression that the process is not effective. Consequently we see that the effect of not taking measurement error into account when it is indeed present can give misleading results understanding effectiveness of the process is in-control. $\bar{X}$-control charts used to compute power curves will usually be subject to measurement error. If this is not taken account statistical inference on the area under the power curve will, as shown in the figures, be seriously misleading in many cases. Not adjusting the measurement in the process control can result in process being overlooked. If the ratio of intra-individual to inter-individual variation is accurately known (based on prior information), a corrected power curve for $\overline{\mathrm{X}}$-control chart can be obtained. Otherwise, it will be necessary to conduct additional experimentation in order to obtain information on the measurement error. Such experiment may be internal with repeated measurements being carried out on some of the process control under consideration. Hence we can conclude that in process control one can take a number of repeated measurements and use their average to represent the true value or 
alternatively look for better measurement techniques that would reduce the measurement. necessary to conduct additional experimentation in order to obtain information on the measurement error. Such experiment may be internal with repeated measurements being carried out on some of the process control under consideration. Hence we can conclude that in process control one can take a number of repeated measurements and use their average to represent the true value or alternatively look for better measurement techniques that would reduce the measurement.

TABLE-1: Power Function of $\bar{X}$-Chart with Known cv under Measurement Error for (n=5)

\begin{tabular}{|c|c|c|c|c|c|c|c|c|c|c|c|c|c|c|c|c|}
\hline \multirow{2}{*}{ d } & \multicolumn{4}{|c|}{$\mathbf{r}=\infty$} & \multicolumn{4}{|c|}{$r=2$} & \multicolumn{4}{|c|}{$r=4$} & \multicolumn{4}{|c|}{$r=6$} \\
\hline & $\mathrm{v}=\mathbf{0}$ & $v=2$ & $v=4$ & $v=6$ & $v=0$ & $v=2$ & $\mathrm{v}=4$ & $v=6$ & $v=0$ & $\mathrm{v}=\mathbf{2}$ & $v=4$ & $v=6$ & $\mathrm{v}=\mathbf{0}$ & $\mathrm{v}=2$ & $\mathrm{v}=4$ & $v=6$ \\
\hline 0.0 & 0.0027 & 0.0001 & 0.0000 & 0.0000 & 0.0073 & 0.0001 & 0.0000 & 0.0000 & 0.0036 & 0.0001 & 0.0000 & 0.0000 & 0.0031 & 0.0001 & 0.0000 & 0.0000 \\
\hline 0.1 & 0.0034 & 0.0001 & 0.0000 & 0.0000 & 0.0085 & 0.0002 & 0.0000 & 0.0000 & 0.0044 & 0.0001 & 0.0000 & 0.0000 & 0.0051 & 0.0001 & 0.0000 & 0.0000 \\
\hline 0.2 & 0.0056 & 0.0003 & 0.0000 & 0.0000 & 0.0122 & 0.0006 & 0.0000 & 0.0000 & 0.0070 & 0.0004 & 0.0000 & 0.0000 & 0.0069 & 0.0003 & 0.0000 & 0.0000 \\
\hline 0.3 & 0.0100 & 0.0009 & 0.0000 & 0.0000 & 0.0191 & 0.0016 & 0.0000 & 0.0000 & 0.0121 & 0.0011 & 0.0000 & 0.0000 & 0.0113 & 0.0010 & 0.0000 & 0.0000 \\
\hline 0.4 & 0.0177 & 0.0024 & 0.0000 & 0.0000 & 0.0301 & 0.0039 & 0.0000 & 0.0000 & 0.0206 & 0.0027 & 0.0000 & 0.0000 & 0.0192 & 0.0025 & 0.0000 & 0.0000 \\
\hline 0.5 & 0.0299 & 0.0058 & 0.0001 & 0.0000 & 0.0463 & 0.0086 & 0.0001 & 0.0000 & 0.0340 & 0.0065 & 0.0001 & 0.0000 & 0.0318 & 0.0061 & 0.0001 & 0.0000 \\
\hline 0.6 & 0.0486 & 0.0130 & 0.0003 & 0.0000 & 0.0691 & 0.0180 & 0.0005 & 0.0000 & 0.0538 & 0.0143 & 0.0004 & 0.0000 & 0.0510 & 0.0136 & 0.0004 & 0.0000 \\
\hline 0.7 & 0.0757 & 0.0271 & 0.0016 & 0.0000 & 0.0997 & 0.0348 & 0.0021 & 0.0000 & 0.0820 & 0.0292 & 0.0018 & 0.0000 & 0.0785 & 0.0280 & 0.0017 & 0.0000 \\
\hline 0.8 & 0.1129 & 0.0521 & 0.0065 & 0.0003 & 0.1394 & 0.0628 & 0.0077 & 0.0003 & 0.1200 & 0.0550 & 0.0069 & 0.0003 & 0.1161 & 0.0534 & 0.0067 & 0.0003 \\
\hline 0.9 & 0.1617 & 0.0926 & 0.0215 & 0.0023 & 0.1886 & 0.1058 & 0.0241 & 0.0026 & 0.1690 & 0.0963 & 0.0222 & 0.0024 & 0.1650 & 0.0943 & 0.0218 & 0.0024 \\
\hline 1.0 & 0.2225 & 0.1527 & 0.0587 & 0.0143 & 0.2472 & 0.1669 & 0.0633 & 0.0154 & 0.2293 & 0.1567 & 0.0600 & 0.0146 & 0.2256 & 0.1545 & 0.0593 & 0.0145 \\
\hline 1.1 & 0.2945 & 0.2343 & 0.1341 & 0.0609 & 0.3145 & 0.2472 & 0.1399 & 0.0632 & 0.3001 & 0.2379 & 0.1358 & 0.0616 & 0.2970 & 0.2359 & 0.1348 & 0.0612 \\
\hline 1.2 & 0.3757 & 0.3355 & 0.2581 & 0.1822 & 0.3885 & 0.3444 & 0.2632 & 0.1852 & 0.3793 & 0.3380 & 0.2596 & 0.1831 & 0.3774 & 0.3366 & 0.2588 & 0.1826 \\
\hline 1.3 & 0.4629 & 0.4503 & 0.4243 & 0.3949 & 0.4668 & 0.4531 & 0.4261 & 0.3961 & 0.4640 & 0.4511 & 0.4249 & 0.3952 & 0.4634 & 0.4507 & 0.4246 & 0.3950 \\
\hline 1.4 & 0.5519 & 0.5695 & 0.6054 & 0.6457 & 0.5465 & 0.5656 & 0.6029 & 0.6440 & 0.5504 & 0.5684 & 0.6047 & 0.6452 & 0.5512 & 0.5690 & 0.6051 & 0.6454 \\
\hline 1.5 & 0.6384 & 0.6826 & 0.7660 & 0.8447 & 0.6243 & 0.6729 & 0.7606 & 0.8417 & 0.6344 & 0.6798 & 0.7644 & 0.8438 & 0.6366 & 0.6814 & 0.7653 & 0.8443 \\
\hline 1.6 & 0.7183 & 0.7808 & 0.8818 & 0.9510 & 0.6973 & 0.7675 & 0.8760 & 0.9489 & 0.7124 & 0.7771 & 0.8801 & 0.9504 & 0.7156 & 0.7791 & 0.8810 & 0.9507 \\
\hline 1.7 & 0.7885 & 0.8588 & 0.9497 & 0.9891 & 0.7632 & 0.8446 & 0.9455 & 0.9883 & 0.7815 & 0.8548 & 0.9485 & 0.9889 & 0.7854 & 0.8570 & 0.9492 & 0.9890 \\
\hline 1.8 & 0.8473 & 0.9154 & 0.9822 & 0.9983 & 0.8203 & 0.9026 & 0.9798 & 0.9981 & 0.8400 & 0.9119 & 0.9815 & 0.9983 & 0.8440 & 0.9138 & 0.9819 & 0.9983 \\
\hline 1.9 & 0.8941 & 0.9530 & 0.9947 & 0.9998 & 0.8679 & 0.9429 & 0.9937 & 0.9998 & 0.8871 & 0.9503 & 0.9945 & 0.9998 & 0.8909 & 0.9518 & 0.9946 & 0.9998 \\
\hline 2.0 & 0.9295 & 0.9759 & 0.9987 & 1.0000 & 0.9060 & 0.9687 & 0.9984 & 1.0000 & 0.9234 & 0.9740 & 0.9986 & 1.0000 & 0.9268 & 0.9750 & 0.9987 & 1.0000 \\
\hline 2.1 & 0.9550 & 0.9885 & 0.9997 & 1.0000 & 0.9353 & 0.9840 & 0.9997 & 1.0000 & 0.9500 & 0.9874 & 0.9997 & 1.0000 & 0.9528 & 0.9880 & 0.9997 & 1.0000 \\
\hline 2.2 & 0.9725 & 0.9950 & 1.0000 & 1.0000 & 0.9570 & 0.9924 & 0.9999 & 1.0000 & 0.9687 & 0.9943 & 1.0000 & 1.0000 & 0.9708 & 0.9947 & 1.0000 & 1.0000 \\
\hline 2.3 & 0.9839 & 0.9980 & 1.0000 & 1.0000 & 0.9724 & 0.9966 & 1.0000 & 1.0000 & 0.9812 & 0.9977 & 1.0000 & 1.0000 & 0.9827 & 0.9978 & 1.0000 & 1.0000 \\
\hline 2.4 & 0.9910 & 0.9993 & 1.0000 & 1.0000 & 0.9829 & 0.9986 & 1.0000 & 1.0000 & 0.9892 & 0.9991 & 1.0000 & 1.0000 & 0.9902 & 0.9992 & 1.0000 & 1.0000 \\
\hline 2.5 & 0.9952 & 0.9997 & 1.0000 & 1.0000 & 0.9897 & 0.9995 & 1.0000 & 1.0000 & 0.9940 & 0.9997 & 1.0000 & 1.0000 & 0.9947 & 0.9997 & 1.0000 & 1.0000 \\
\hline
\end{tabular}

TABLE-2: Power Function of $\bar{X}$-Chart with Known cv under Measurement Error for (n=7)

\begin{tabular}{|c|c|c|c|c|c|c|c|c|c|c|c|c|c|c|c|c|}
\hline \multirow{2}{*}{ d } & \multicolumn{4}{|c|}{$\mathbf{r}=\infty$} & \multicolumn{4}{|c|}{$r=2$} & \multicolumn{4}{|c|}{$r=4$} & \multicolumn{4}{|c|}{$r=6$} \\
\hline & $\mathrm{v}=\mathbf{0}$ & $\mathrm{v}=2$ & $\mathrm{v}=4$ & $\mathrm{v}=6$ & $v=0$ & $v=2$ & $v=4$ & $v=6$ & $v=0$ & $v=2$ & $\mathrm{v}=\mathbf{4}$ & $v=6$ & $v=0$ & $v=2$ & $\mathrm{v}=4$ & $\mathrm{v}=6$ \\
\hline 0.0 & 0.0027 & 0.0002 & 0.0000 & 0.0000 & 0.0073 & 0.0004 & 0.0000 & 0.0000 & 0.0036 & 0.0002 & 0.0000 & 0.0000 & 0.0031 & 0.0002 & 0.0000 & 0.0000 \\
\hline 0.1 & 0.0037 & 0.0003 & 0.0000 & 0.0000 & 0.0090 & 0.0007 & 0.0000 & 0.0000 & 0.0048 & 0.0004 & 0.0000 & 0.0000 & 0.0052 & 0.0004 & 0.0000 & 0.0000 \\
\hline 0.2 & 0.0069 & 0.0010 & 0.0000 & 0.0000 & 0.0144 & 0.0019 & 0.0000 & 0.0000 & 0.0086 & 0.0012 & 0.0000 & 0.0000 & 0.0082 & 0.0011 & 0.0000 & 0.0000 \\
\hline 0.3 & 0.0138 & 0.0028 & 0.0000 & 0.0000 & 0.0246 & 0.0049 & 0.0001 & 0.0000 & 0.0163 & 0.0033 & 0.0000 & 0.0000 & 0.0151 & 0.0031 & 0.0000 & 0.0000 \\
\hline 0.4 & 0.0261 & 0.0075 & 0.0002 & 0.0000 & 0.0414 & 0.0115 & 0.0003 & 0.0000 & 0.0298 & 0.0085 & 0.0002 & 0.0000 & 0.0279 & 0.0079 & 0.0002 & 0.0000 \\
\hline 0.5 & 0.0468 & 0.0178 & 0.0012 & 0.0000 & 0.0669 & 0.0248 & 0.0016 & 0.0000 & 0.0519 & 0.0196 & 0.0013 & 0.0000 & 0.0491 & 0.0186 & 0.0012 & 0.0000 \\
\hline
\end{tabular}




\begin{tabular}{|c|c|c|c|c|c|c|c|c|c|c|c|c|c|c|c|c|}
\hline 0.6 & 0.0789 & 0.0383 & 0.0052 & 0.0002 & 0.1032 & 0.0490 & 0.0065 & 0.0003 & 0.0853 & 0.0412 & 0.0056 & 0.0002 & 0.0818 & 0.0396 & 0.0054 & 0.0002 \\
\hline 0.7 & 0.1255 & 0.0751 & 0.0187 & 0.0022 & 0.1523 & 0.0894 & 0.0219 & 0.0026 & 0.1327 & 0.0790 & 0.0196 & 0.0023 & 0.1287 & 0.0768 & 0.0191 & 0.0023 \\
\hline 0.8 & 0.1885 & 0.1341 & 0.0547 & 0.0143 & 0.2147 & 0.1504 & 0.0604 & 0.0156 & 0.1957 & 0.1386 & 0.0563 & 0.0147 & 0.1918 & 0.1361 & 0.0554 & 0.0145 \\
\hline 0.9 & 0.2680 & 0.2189 & 0.1310 & 0.0625 & 0.2900 & 0.2343 & 0.1385 & 0.0657 & 0.2741 & 0.2233 & 0.1331 & 0.0635 & 0.2708 & 0.2209 & 0.1320 & 0.0630 \\
\hline 1.0 & 0.3616 & 0.3285 & 0.2604 & 0.1900 & 0.3757 & 0.3391 & 0.2669 & 0.1939 & 0.3656 & 0.3315 & 0.2623 & 0.1911 & 0.3634 & 0.3299 & 0.2613 & 0.1905 \\
\hline 1.1 & 0.4643 & 0.4552 & 0.4354 & 0.4121 & 0.4680 & 0.4582 & 0.4374 & 0.4135 & 0.4653 & 0.4561 & 0.4360 & 0.4125 & 0.4648 & 0.4556 & 0.4357 & 0.4122 \\
\hline 1.2 & 0.5694 & 0.5868 & 0.6244 & 0.6677 & 0.5622 & 0.5811 & 0.6207 & 0.6651 & 0.5674 & 0.5852 & 0.6233 & 0.6669 & 0.5685 & 0.5860 & 0.6239 & 0.6673 \\
\hline 1.3 & 0.6698 & 0.7092 & 0.7872 & 0.8620 & 0.6529 & 0.6966 & 0.7799 & 0.8580 & 0.6651 & 0.7056 & 0.7851 & 0.8608 & 0.6677 & 0.7075 & 0.7862 & 0.8614 \\
\hline 1.4 & 0.7593 & 0.8113 & 0.8991 & 0.9595 & 0.7356 & 0.7952 & 0.8919 & 0.9569 & 0.7527 & 0.8067 & 0.8970 & 0.9588 & 0.7563 & 0.8092 & 0.8981 & 0.9592 \\
\hline 1.5 & 0.8336 & 0.8877 & 0.9604 & 0.9918 & 0.8068 & 0.8717 & 0.9556 & 0.9909 & 0.8263 & 0.8832 & 0.9591 & 0.9916 & 0.8303 & 0.8856 & 0.9598 & 0.9917 \\
\hline 1.6 & 0.8912 & 0.9389 & 0.9873 & 0.9989 & 0.8650 & 0.9257 & 0.9849 & 0.9987 & 0.8842 & 0.9353 & 0.9866 & 0.9988 & 0.8881 & 0.9373 & 0.9870 & 0.9989 \\
\hline 1.7 & 0.9329 & 0.9698 & 0.9967 & 0.9999 & 0.9098 & 0.9603 & 0.9957 & 0.9999 & 0.9269 & 0.9673 & 0.9964 & 0.9999 & 0.9302 & 0.9686 & 0.9966 & 0.9999 \\
\hline 1.8 & 0.9610 & 0.9864 & 0.9993 & 1.0000 & 0.9425 & 0.9805 & 0.9990 & 1.0000 & 0.9563 & 0.9849 & 0.9992 & 1.0000 & 0.9589 & 0.9857 & 0.9993 & 1.0000 \\
\hline 1.9 & 0.9787 & 0.9945 & 0.9999 & 1.0000 & 0.9651 & 0.9912 & 0.9998 & 1.0000 & 0.9754 & 0.9937 & 0.9999 & 1.0000 & 0.9772 & 0.9941 & 0.9999 & 1.0000 \\
\hline 2.0 & 0.9890 & 0.9980 & 1.0000 & 1.0000 & 0.9798 & 0.9964 & 1.0000 & 1.0000 & 0.9869 & 0.9976 & 1.0000 & 1.0000 & 0.9881 & 0.9978 & 1.0000 & 1.0000 \\
\hline 2.1 & 0.9947 & 0.9993 & 1.0000 & 1.0000 & 0.9889 & 0.9986 & 1.0000 & 1.0000 & 0.9934 & 0.9992 & 1.0000 & 1.0000 & 0.9942 & 0.9993 & 1.0000 & 1.0000 \\
\hline 2.2 & 0.9976 & 0.9998 & 1.0000 & 1.0000 & 0.9942 & 0.9995 & 1.0000 & 1.0000 & 0.9969 & 0.9997 & 1.0000 & 1.0000 & 0.9973 & 0.9998 & 1.0000 & 1.0000 \\
\hline 2.3 & 0.9990 & 0.9999 & 1.0000 & 1.0000 & 0.9971 & 0.9998 & 1.0000 & 1.0000 & 0.9986 & 0.9999 & 1.0000 & 1.0000 & 0.9988 & 0.9999 & 1.0000 & 1.0000 \\
\hline 2.4 & 0.9996 & 1.0000 & 1.0000 & 1.0000 & 0.9986 & 1.0000 & 1.0000 & 1.0000 & 0.9994 & 1.0000 & 1.0000 & 1.0000 & 0.9995 & 1.0000 & 1.0000 & 1.0000 \\
\hline 2.5 & 0.9998 & 1.0000 & 1.0000 & 1.0000 & 0.9994 & 1.0000 & 1.0000 & 1.0000 & 0.9998 & 1.0000 & 1.0000 & 1.0000 & 0.9998 & 1.0000 & 1.0000 & 1.0000 \\
\hline
\end{tabular}
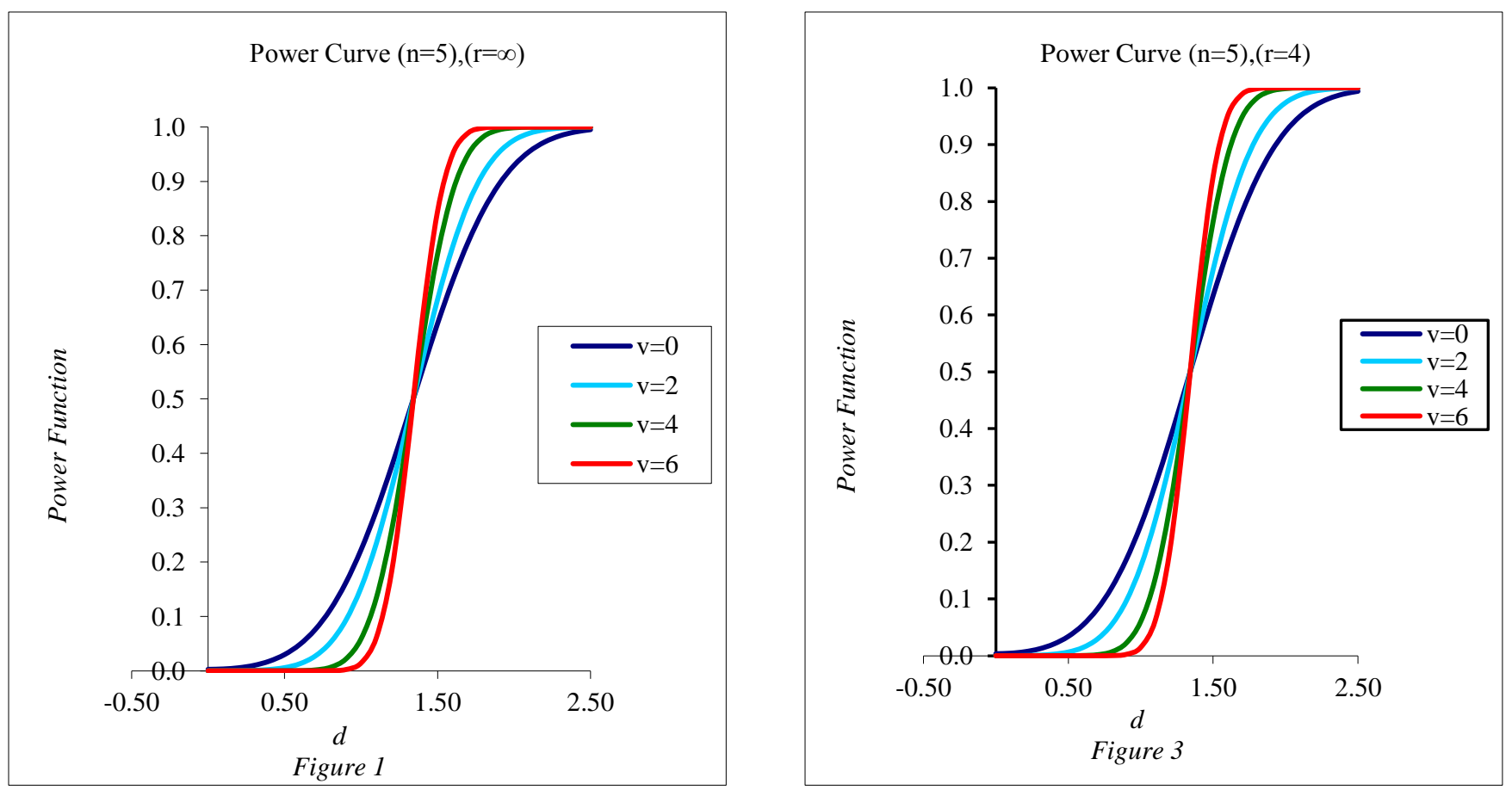

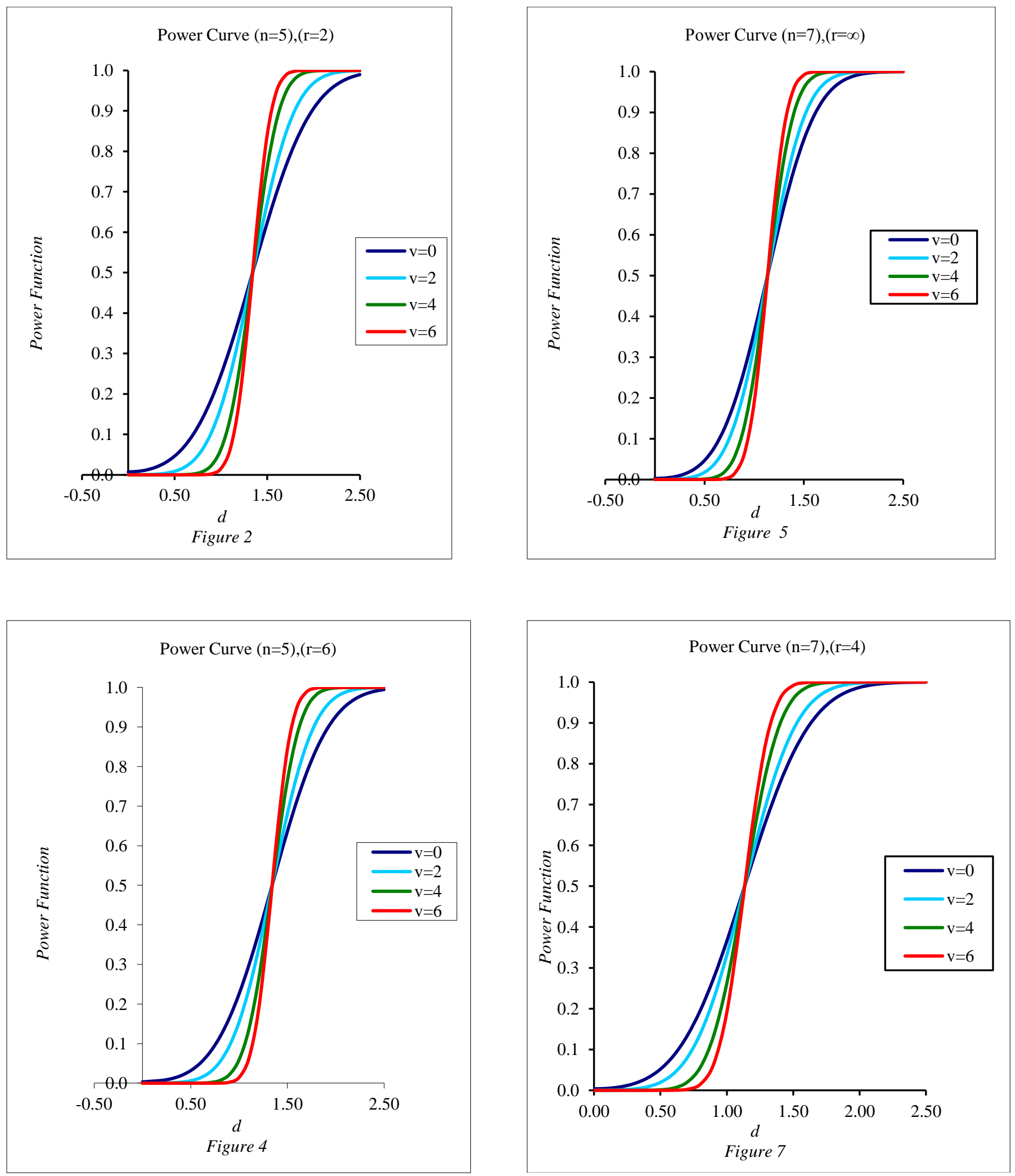

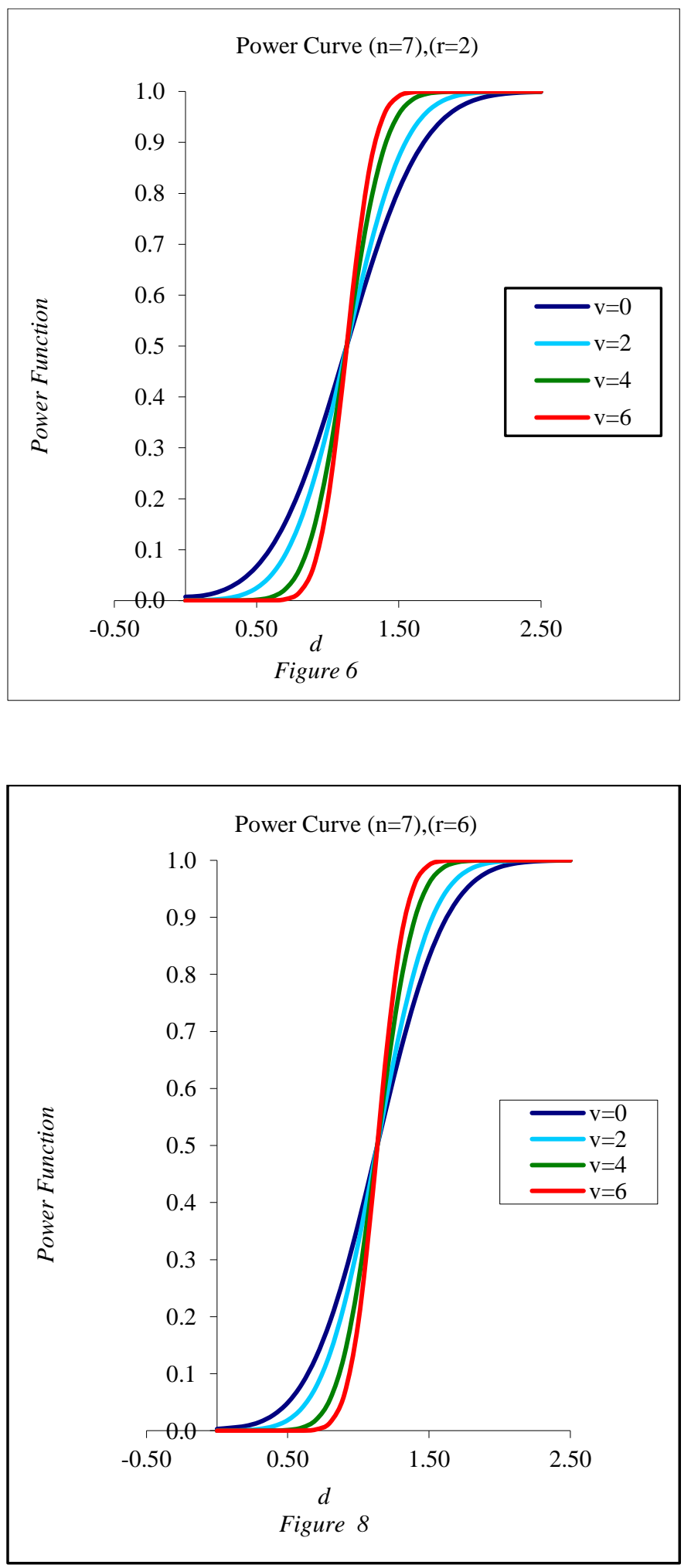

\section{Reference}

[1] Automotive Industry Action Group, Chrysler, Ford, GM, Measurement Systems Analysis, AIAG, Detroit, MI, 1995.

[2] K. Linna and W.H.Woodall, Effect of measurement error on shewhart control charts, J. Qual. Technol. 33(2) (2001), pp. 213-222.

[3] K. Linna and W.H.Woodall, The performance of multivariate control charts in the presence of measurement error, J. Qual. Technol. 33(3) (2001), pp. 349-355.

[4] P.E. Maravelakis, J. Panaretos, and S. Psarakis, EWMA chart and measurement error, J. Appl. Stat. 31(4) (2004), pp. 445455.

[5] D.C. Montgomery, Statistical Process Control, JohnWiley \& Sons, NewYork, 2009.

[6] D.C. Montgomery and G.C. Runger, Gauge capability and designed experiments. Part 1: Basic methods, Qual. Eng. 6(1) (1993), pp. 115-135.

[7] M.A. Rahim, Economic model of $X$ charts under nonnormality and measurement errors, Int. J. Comput. Opera. Res. 9(3) (1985), pp. 291-299.

[8] D. T. Searls, The utilization of a known coefficient of variation in the estimation procedure, Journal of the American Statistical Association, 59,( 1964), pp. 1225-1226. 